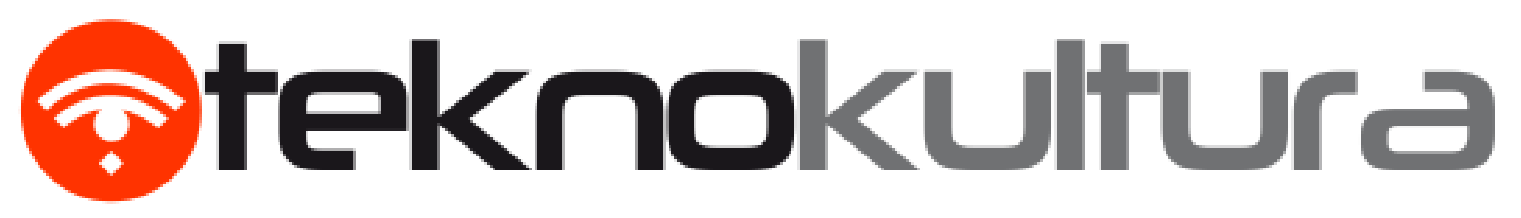

\#Toret Medina, J. (2015). Una mirada tecnopolítica al primer año de Podemos. Seis hipótesis. Revista Teknokultura, Vol. $12(1), 121-135$.

Recibido: 27-03-2015

Aceptado: 07-04-2015
Open peer review

http://revistas.ucm.es/index.php/TEKN/pages/view/opr-276

\title{
Una mirada tecnopolítica al primer año de Podemos Seis hipótesis
}

\section{A techno-political look at the first year of Podemos Six Hypotheses}

\author{
Javier Toret Medina \\ Universitat Oberta de Catalunya \\ toret9@gmail.com
}

\section{Resumen}

La irrupción de Podemos en las elecciones europeas de mayo de 2014 ha transformado radicalmente la escena política española. El impacto de Podemos se enmarca en un contexto de crisis económica, creciente intolerancia contra la corrupción y una paisaje cultural en transformación tras los múltiples efectos del movimiento-red 15M. En este breve ensayo se trata de poner de relieve algunos aspectos y dar fuerza a miradas que muestran a Podemos atendiendo a su conexión con los Adn del 15M y latinoamericano, como su estratégica de ocupación de los medios de comunicación de masas como el de las redes sociales digitales. Atendemos al crecimiento exponencial y una mirada que muestra elementos determinantes menos visibles en la emergencia política y comunicativa de Podemos. 


\section{Palabras Clave}

Podemos, 15M, comunicación multicapa, crecimiento exponencial, front-end, back-end.

\section{Abstract}

The eruption of Podemos in 2014 has radically transformed the Spanish political scene. Its impact can be framed in a context of economic crisis, increasing intolerance towards corruption and a changing cultural landscape after the effects of the $15 \mathrm{M}$ network-movement. In this essay we highlight some aspects of Podemos in relation to its $15 \mathrm{M}$ and Latin-American ADN, such as its strategic occupation of mass media and digital social networks. We look at Podemos exponential growth by focusing on less visible but key elements of its political and communicative emergence.

\section{KeYWORDS}

Podemos, 15M, multilayer communication, exponential growth, front-end, back-end

\section{Sumario}

Podemos no se entiende sin el 15M: Primera Hipótesis

Podemos no se explica solo por el 15M. El ADN latinoamericano, un marco conceptual largamente construido: Segunda Hipótesis

\#OccupyTheMedia: La Tuerka y la toma de los platós hacia \#OccupyTheState:

\section{Tercera Hipótesis}

Año 1 : crecimiento acelerado y exponencial de Podemos (tras 25M): Cuarta Hipótesis

Podemos como circuito optimizado de comunicación multicapa: Quinta Hipótesis

Pensar Podemos como un front end y back end: Sexta Hipótesis

Recapitulación

Referencias 


\section{SUMmaRY}

Podemos cannot be explained only by $15 \mathrm{M}$. Latin American DNA, a largely built conceptual framework: Second Hypothesis

\#OccupyTheMedia: From La Tuerka and the taken on of tv set to \#OccupyTheState:

Third Hypotheses

Year One: Podemos exponential growth (after 25M): Fourth Hypotheses

Podemos as optimized communication multilayer circuit: Fifth Hypotheses

Podemos front-end and back-end: Sixth Hypothesis

Recapitulation

References 

La irrupción de Podemos en 2014 ha transformado radicalmente la escena política española. Los cinco diputados y 1.245 .908 votos conseguidos en tan solo cuatro meses de vida de la formación, convirtiéndose en la 4º fuerza más votada del país, no reflejan únicamente un dato cuantitativo de los resultados de las elecciones europeas. La fulgurante aparición electoral de un proceso masivo de participación y comunicación de gran impacto en la esfera pública, que se puso como objetivo convertir la indignación en poder político-institucional, es el aspecto cualitativo de transformación del campo político. El impacto de Podemos se enmarca en un contexto de crisis económica, en una creciente intolerancia contra la corrupción y una paisaje cultural en transformación tras los múltiples efectos del movimiento-red 15M.

\section{Podemos no se entiende sin el 15M: Primera Hipótesis}

Podemos nace en un contexto social marcado por un efecto capilar de los acontecimientos desencadenados desde el 15 de Mayo del 2011, tras la manifestación convocada por Democracia Real Ya en más de 60 ciudades, y las acampadas que se extendieron por todo el país. 2012 fue el año con más manifestaciones en España: 46.000 manifestaciones, 120 al día.

El 7 de abril de 2011 Juventud Sin Futuro protagonizó una movilización en Madrid, antesala de la movilización del 15M en mayo. En este punto hay que destacar que una parte de los activistas de Juventud Sin Futuro fueron integrantes de Podemos desde sus inicios. Como consecuencia de su experiencia previa, el manejo de la comunicación en red y de las claves comunicativas del contexto post $15 \mathrm{M}$, son capacidades controladas por los equipos de redes que lanzan y promocionan Podemos.

El lanzamiento se realizó en un contexto donde el 15M había apuntalado la ya baja credibilidad de los partidos, sindicatos y otras instituciones; algunos lo han llamado "crisis de régimen" y-o "situación populista". A su vez, Podemos se subió a la ola de gran legitimidad del propio $15 \mathrm{M}$ y de las plataformas ciudadanas como la Plataforma de Afectados por la Hipoteca o las distintas mareas ciudadanas.

En palabras de Pablo Iglesias, Podemos asume desde un primer momento "el cambio del tablero, de las coordenadas de la política" (Rivero, 2014) que supuso el 15M. En contraposición al 
extrañamiento e incomprensión de la izquierda institucionalizada, el equipo promotor se reconoce en el diagnóstico del 15M: situarse más allá del eje izquierda-derecha y la crítica a la alianzacomplicidad entre intereses de la banca y los partidos políticos, para después asumir el cambio cultural que supone el $15 \mathrm{M}$. Aunque las formas organizativas que se plantean no son las mismas que las del repertorio $15 \mathrm{M}$, se extraen formas y haceres comunicativos, así como símbolos surgidos del ecosistema $15 \mathrm{M}$, muchas veces sin reconocerlo explícitamente.

Podemos articula y moviliza el malestar y un nuevo sentido común que surgen a partir del impacto que generó tanto el acontecimiento como el proceso de politización masiva del 15M. Las encuestas publicadas en 2011 mostraron que en el $1^{\mathbf{o}}$ año del $15 \mathrm{M}$ participaron aproximadamente 8.500 .000 de personas, la gran mayoría sin pertenencia a ninguna organización social previa.

En el 15M están "los políticos y banqueros" como un afuera genérico y difuso. La lógica de creación de una identidad colectiva común reside en las múltiples formas de participación, de "formar parte de", en su carácter abierto, difuso, dinámico y reapropiable. Podemos genera un marco de identificación común mucho más definido, si bien, hereda parte de esta construcción de la identidad abierta y reapropiable, como se observa en sus inicios, a través de la multiplicación de los círculos y de prácticas tecnopolíticas a través del uso intensivo de las redes de comunicación para la acción colectiva. Podemos recoge parte de los frutos que fueron sembrados en el 15M, así como las luchas sociales y populares de años anteriores. La "innovación y capacidad" de catalizar y dar forma a este malestar se orientan a su constitución como poder político, en un discurso que produce hegemonía, en un poder que se quiere constituyente frente al "régimen del 78".

\section{Podemos no se explica solo por el $15 \mathrm{M}$ EI ADN latinoamericano, un marco conceptual largamente construido: Segunda Hipótesis}

El saber-hacer del 15M y el conocimiento depurado de la experiencia en campañas electorales y gobiernos de cambio en América latina (en Bolivia, Argentina, Venezuela y Ecuador) produjo una combinación explosiva. Esta extraña combinación del know-how del $15 \mathrm{M}$ y de la experiencia latinoamericana son centrales para el éxito del lanzamiento y la viabilidad de la iniciativa. 
No obstante, y a diferencia del 15M, Podemos surge de una hipótesis largamente elaborada, bajo una perspectiva teórica gramsciana-laclauliana. Esta hipótesis, que se apoya en la teoría del discurso y el análisis de marcos adaptados, contextualizados y sintetizados, como queda reflejado en la tesis doctoral de Iñigo Errejón sobre la función del discurso en la producción de hegemonía en Bolivia. A este respecto Errejón plantea lo siguiente:

Sin ideas, ni un marco conceptual, lo que hacemos es acumular experiencias que caducan al día siguiente. Es fundamental el trabajo intelectual. Cualquiera que nos conozca un poco sabe que en Podemos hay un hipótesis intelectual largamente larvada, largamente discutida. Uno puede ver haciendo hemeroteca o revisión de bibliografía, que hay todo un trabajo de elaboración, discusión y análisis de prueba de hipótesis, un trabajo modesto que se encontró con unas circunstancias favorables $[\ldots]$

(Errejón, 2015)

En realidad la idea de Podemos o sus raíces intelectuales son profundas, aunque poco visibles como corresponde al trabajo académico. A pesar de que no se trata de una construcción de laboratorio, la base de Podemos la componen ideas en las que venimos trabajando desde hace tiempo a través de un grupo muy pequeño de personas en la Facultad de Ciencias Políticas y Sociología de la Universidad Complutense de Madrid. Para nosotros se trata de atreverse a lanzar una hipótesis y de trabajar en desarrollarla. ${ }^{1}$

(Errejón, 2015)

Especialmente interesante es la combinación de conocimiento teórico-académico de los promotores de Podemos con su traducción en la experiencia sobre la centralidad de comunicación política, vinculada al desarrollo del debate y la disputa política en los grandes medios de comunicación de masas. Como ha reconocido Pablo Iglesias en numerosas ocasiones: "Podemos no hubiera sido posible sin La Tuerka".

Podemos como práctica cultural emergente frente al imaginario neoliberal: hegemonía y disidencia. Conversación con Íñigo Errejón:

<https://ipena44.files.wordpress.com/2014/12/errejon_claves.pdf> 


\section{\#OccupyTheMedia:}

\section{La Tuerka y la toma de los platós hacia \#OccupyTheState: Tercera Hipótesis}

De los movimientos sociales, de los espacios académicos y de una televisión de muy bajo presupuesto, al centro de la atención mediática en programas máxima audiencia; de que te escuchen o lean cientos de personas, a que lo hagan millones. Un recorrido meteórico que tiene que ver con una visión estratégica de la centralidad de los medios de (configuración) comunicación en la construcción de los argumentarios, posiciones, identidades e interpretaciones sobre la realidad. La construcción de un estilo de conversación, de debates adecuados a los formatos televisivos, de un saber hacer comunicacional y la producción de discurso público para el gran público, todos ellos son elementos clave de la estrategia que ha permitido acumular capital simbólico en torno a Podemos. Atravesar y ocupar el espacio mediático ha sido un elemento central para Pablo Iglesias desde hace años, una cuestión en la que ha insistido repetidamente aseverando que "el espacio principal de socialización de la gente son los medios de comunicación de masas, especialmente: la televisión" (Rivero, 2014). Por lo tanto, se implementan estrategias enfocadas a aprovechar este espacio:

"Lo que mucha gente no se imagina es que nuestra intervención en los medios era una cosa muy reflexionada, llevábamos años preparándonos para eso [...] La Tuerka fue el espacio donde empezamos a aterrizar, en el que convertimos nuestros diagnósticos y nuestras hipótesis en argumentos y en arma para el combate de las tertulias"

(Rivero, 2014)

Desde un programa al estilo de 59 Segundos, realizado en la universidad Complutente en 2009, a un programa en una televisión de barrio como Tele $K$ en 2010 --con un canal activo YouTube y con buena circulación en redes-- al paso por el denominado "TDT Party" en abril 2013, se produce el salto a las tertulias de televisión estatales generalistas y a programas de notable audiencia como La Sexta Noche y Las Mañanas Cuatro.

Se trata de aprovechar las rendijas y las paradojas del espacio mediático privado para lanzar un mensaje claro, que con un lenguaje directo y muy preparado, interpela y conmueve a un público mayoritario, que tas el $15 \mathrm{M}$ está ávido de posiciones fuera del establishment. Los 
políticos de los partidos afincados en el poder están en los medios, sin embargo, siguen sin generar procesos de identificación e ilusión. Desde que Iglesias participara en abril de 2013 en el programa El Gato Al agua (InterconomíaTv) - "cruzando las líneas enemigas", en sus propias palabras - se embarca en múltiples batallas de confrontación argumental donde la audiencia se incrementaban de manera considerable. Esté "paso" le proporciona gran visibilidad, reputación y seguidores en las redes sociales.

Después de las elecciones Pablo Iglesias siguió revolucionando las audiencias. Hace crecer la cuota de pantalla de los programas Las Mañanas de Cuatro y La Sexta Noche al tiempo que multiplica su popularidad personal. La audiencia media de Las Mañanas de Cuatro en 2013 era del 5,4\%, en 2014 fue del 7,8\% hasta la irrupción de Podemos. Desde las elecciones europeas, no bajó del $10 \%$ de share. La semana posterior del 25 de mayo tuvo una cuota media del 11,6\%. El programa alcanzó su récord con el 12,8\% de share, una cuota a la que se llega bien con Pablo Iglesias como tertuliano en plató, bien mediante una conexión en directo. Durante los días 26 y 27 de mayo de 2014, la aparición de Iglesias se convierte en el minuto de oro con una cuota de $14,4 \%$.

A la inteligencia del equipo de La Tuerka y su toma del espacio mediático, se suma al interés de las televisiones privadas por incrementar su share. El aumento de la audiencia televisiva y, en consecuencia, de la popularidad de Pablo Iglesias, generan un círculo virtuoso de popularidad que le sitúan como figura política emergente en 2013. No obstante, el salto exponencial se produce a partir del 25 de mayo, tras las elecciones europeas.

\section{Año 1 : crecimiento acelerado y exponencial de Podemos (tras 25M): Cuarta Hipótesis}

La gran irrupción mediática de Podemos conectó con miles personas. Precipitó tiempos vertiginosos provocados por la velocidad de agregación, enganche, ilusión y soporte de la iniciativa. En su presentación en el Teatro Latina el 14 enero de 2014 —explicada horas más tarde en directo en Las Mañanas de Cuatro-, Podemos pidió el apoyo de 50.000 personas para poder "mover ficha". En menos de 48 horas se consiguieron las firmas. El crecimiento tanto en Twitter como en Facebook fue significativo. En los tres meses previos a la campaña, Podemos articuló una masa crítica activa y una la proliferación rápida y extensa de sus círculos a través de las principales redes sociales (agrupaciones territoriales y sectoriales base de la organización). 
Semejante crecimiento se produce gracias a un modelo de comunicación multicapa (Toret, 2015) optimizado con un crecimiento extenso en lo territorial, con los círculos. Este crecimiento también se produce en lo mediático, con audiencias televisivas importantes y continuas, y en lo digital, con la creación acelerada y emergente de un ecosistema de perfiles en redes sociales con gran número de seguidores. Esta hibridación permitió un desborde participativo con el ímpetu propio de un movimiento red, pero también con el encuadre político, estratégico y discursivo de un think tank político.

En esos cuatro meses se construyeron 400 círculos donde participaron cotidianamente miles de personas. En un año se llega a 900 círculos dentro y fuera del país. Todos ellos de pertenencia totalmente abierta a la participación. Los círculos resultaron un elemento esencial del combustible en el territorio y en la campaña. Al mismo tiempo creció el número de sus seguidores en redes sociales: desde el 25 mayo y en una semana, de 200.000 seguidores en Facebook se pasó a 610.000 , y de 60.000 en Twitter, se pasó a 200.000. Un pico de crecimiento parecido a los experimentados en el estallido del 15M.

Tras el 25 de mayo de 2014, Pablo Iglesias superó a Mariano Rajoy en Twitter. Por su parte, Podemos supera de largo a PSOE y PP en la misma red social, y en Facebook la diferencia es aún más notable. Este crecimiento y participación masiva queda a su vez reflejado en las votaciones internas del partido. Durante las primarias las votaciones digitales organizadas en la web participa.podemos.info y gestionadas por la consultora especializadas en voto encriptado Agora Voting) durante las primarias para las europeas con más de 30.000 votos. En la asamblea constituyente (10-14 de noviembre de 2014), La Asamblea Ciudadana Sí Se Puede, 107.488 personas votaron la composición de los órganos de dirección estatales de Podemos. Los documentos Ético, Político y Organizativo, código fuente de la formación, fueron votados por 112.070 personas entre el 20 y 26 de octubre de 2014.

Podemos tuvo un gran impacto desde su lanzamiento, si bien, como hemos apuntado, experimenta un crecimiento exponencial tras los resultados de las elecciones europeas. Podemos nació como ataque relámpago con una forma de desbordamiento similar al que construyó Democracia Real Ya en la campaña del 15 de mayo. Aunque en la Asamblea Sí Se Puede Podemos empieza un proceso de transformación que incluye una estructura reglada, con raíces en el territorio y que dota de órganos y un código constituyente al proceso. Una fuerza que péndula con elementos de movimiento en red con la forma de un partido emergente-populista y que se estructura en torno a un circuito optimizado multicapa de comunicación. 


\section{Podemos como circuito optimizado de comunicación multicapa: Quinta Hipótesis}

Podemos ha sido capaz de construir una organización a partir de un circuito de comunicación integrada. La interacción transmedia, entre el crecimiento territorial, digital y mediático, suponen un modelo de comunicación multicapa optimizado (respecto al del 15M, ya que domina con mayor control la esfera televisiva). Podemos ha puesto en marcha la producción y circulación masiva de un discurso crítico, una activación emocional-pasional colectiva para la participación social y digital masiva, una "máquina de guerra electoral”, como decía Errejón. ${ }^{2}$

El circuito de comunicación ampliado de Podemos genera un gran impacto social partiendo de la articulación de miles de procesos sociales de distintas escalas, en distintos canales y espacios. Esto puede percibirse y describirse con claridad atendiendo a las múltiples capas de comunicación-participación donde Podemos está presente: Web, Facebook, Twitter, Plaza Podemos, Appgree, Loomio, Participa-inscritos, presencia en prensa, radio, televisión. En cada una de estas capas supera el impacto del PP y PSOE. Su base social está más digitalizada y es más participativa que el resto de partidos políticos en España. Podemos se mueve entre distintos espacios y canales, lo que les permite generar "un todo conectado", un circuito multicapa de comunicación.

En el ranking de webs en España, Podemos ocupa el lugar 634, el PSOE el 4.410 y el PP el 11.127. El tiempo que pasan en la web de Podemos $(5: 12,26 \%)$ sus visitantes es el doble que el que pasan los visitantes de las páginas del PP (2:11, 4\%) y del PSOE (2:51, 5\%). Ningún partido cuenta con un espacio tan amplio y diversificado como tiene Podemos en la red social con más usuarios en España. El perfil central de Podemos en Facebook es el más grande de todos los partidos: 937.000. El ecosistema de sus fanpages es muy extenso y cuenta con más de 5.000 seguidores, lo constituyen un total de 100 fanpages con más de 3.000 "me gusta". ${ }^{3}$ Un nodo central, con una red amplia y cierto tamaño de nodos territoriales y temáticos.

A la masiva presencia de Podemos en las redes sociales hegemónicas, se suma el alto nivel de digitalización de sus 900 círculos. Muchos de ellos se han organizado digitalmente

\footnotetext{
${ }^{2}$ Iñigo Errejón en publico. Disponible en <http://www.guerrillerosglobales.com/politica/inigo-errejon-vamos-a-construir-una-maquinaria-de-guerra-electoral/>

3 Datos por consulta propia de Enero de 2015. Con $<\mathrm{http}: / /$ www.alexa.com/ y http://www.fanpagekarma.com/>
} 
e internamente con Loomio - la herramienta australiana que desarrollaron personas cercanas al movimiento Occupy y que permite el trabajo en grupos, y un sistema de propuestas y acuerdos-. Además, podemos uso y popularizó la aplicación Appgree, usada durante su asamblea ciudadana para interaccionar desde el público con los distintos equipos y propuestas.

Otra capa innovadora de Podemos es Plaza Podemos, basada en el software Reddit. Plaza Podemos construyó un enorme ágora sobre la actualidad de Podemos, una plaza digital con vida colectiva que piensa, debate y coopera socializando debates e información y generando procesos de inteligencia colectiva. En octubre de 2014, Plaza Podemos alcanzó un récord con 2.675.000 páginas vistas y 279.285 usuarios únicos.

La participación en estos foros es el principal activo para el crecimiento de Podemos como una masa crítica y participativa. Sus participantes invierten mucho tiempo, a ritmos distintos y en distintos canales, lo cual permite: a) la producción y el hackeo de la opinión publica, así como el re-encuadre de los debates y campos de disputa semántica-significante; b) la movilización de la organización, sincronizándola continuamente con foco de atención; c) la posibilidad de articular de forma frecuente procesos masivos de difusión de información.

Podemos se hace en la comunicación y es una máquina de comunicación. Sus dispositivos de comunicación y capas digitales-sociales han construido la organización. No se trata de una organización con una forma de comunicación, sino una comunicación que desencadena un proceso social con millones de interacciones y conexiones que permiten construir a Podemos como organización.

\section{Pensar Podemos como un front end y back end: Sexta Hipótesis}

Front end o interfaz se entiende en lenguaje de programación como la parte frontal del sistema, lo que ves, lo que es más visible. El front end de Podemos son sus portavoces, lo más visible y mediático, con experiencia en comunicación y debate. Los portavoces que recurren a sus conocimientos y técnicas para posicionar los temas, para contestar a sus adversarios políticos, para usar el discurso como forma de disputar hegemonía y de este modo enmarcar los temas apropiándose de significantes clave. Se trata de un dispositivo de producción de discurso que tiene como centro la primacía audiovisual, así como el rostro de los dirigentes o portavoces. Esta parte de la organización tiene la máxima visibilidad.

El back end o motor es la parte invisible de los procesos que permite que la organización de una web funcione, es el soporte del front end, invisible desde fuera. En el caso de 
Podemos, los círculos son poco visibles desde la perspectiva mediática, a pesar de ser la unidad básica de la organización en su inicio, donde se nutre Podemos en sus procesos territoriales. Son el músculo de la organización. Aunque también lo es la masa crítica que participa en su diversas capas digitales antes descritas.

El back end también permite la estructura de participación y el funcionamiento de la capas de comunicación de la organización. En Podemos el portal participa.podemos.info (de desarrollo propio en software libre ), integrado con Agora Voting, permite que las más de 300.000 personas inscritas participen en las primarias abiertas obligatorias en la organización para la conformación de listas electorales y de los órganos internos de Podemos. Aquí hay un debate sobre cuanto deben ser vinculantes o no la participación masiva de los miembros de podemos en la decisiones estratégicas, y si los modelos de construcción de listas son los más justos o no.

Es destacable dentro de la función del back end encargada al equipo de particiación que incluye la colaboración del grupo LaboDemo, compuesto de técnicos expertos en participación digital, programadores, y que fue una parte muy activa en el 15M. para extender la participación y, de este modo, abrir la organización más allá de sus militantes más comprometidos. Aquí, la tecnología es un aliado perfecto para que las personas 'sientan suyo el proceso y la organización y puedan participar a través de los distintos canales, sin que ello elimine las tensiones políticas sobre como hay que orientar los procesos de participación.

Así pues, en el back end de Podemos se incluye todo el trabajo invisible, desde el montaje y puesta en escena de los actos públicos, hasta la gestión de la participación, de las redes sociales o la producción de argumentarios. Mirar solo el front end de la organización supondría una falta de comprensión de la dimensión masiva de participación social e ilusión que despierta Podemos en franjas amplias de la población española, como han demostrado los actos, mítines o los procesos de participación digital. Supondría, a su vez, infravalorar las dimensiones estructurales del proyecto: Podemos es la gente que lo sostiene, en la medida que hay una masa crítica que aporta en la financiación a través de las campañas de crowdfunding, eligen a los candidatos en las votaciones, mantiene vivo los múltiples canales y procesos de base en Podemos.

De alguna forma, el front-end de Podemos es una máquina de crear movilización e ilusión en esa gran caja de resonancia del sistema social post-15M, y lo interesante es que el back-end no se construye ni de una forma vertical ni de una forma democrática en términos clásicos (por eje. la estructura de IU), sino como resultado de esos procesos de resonancia a partir del front-end que sean capaces de construir: desde los círculos que sólo funcionan si son capaces de emerger y aglutinar gente de una forma muy parecida a los procesos 15M, Esto es extraño en términos democráticos clásicos (tanto representativos como asamblearios), pero consigue 
crear una máquina de dispositivos especializados en construir un front-end capaz de activar adecuadamente esa caja de resonancia post-15M, es decir, en generar procesos de ilusión y participación masivos (Aguilera 2015).

Esta tensión entre un front end y back end que por una lado están resonando y parcialmente desacoplados (para que cada uno realice su función) tiene que 'estar en sintonía' para el modelo funcione, si no es así los limites del proyecto pueden resentirse.

\section{Recapitulación}

La re-activación de las pasiones alegres de la política que despertó el 15M y que encarnó la PAH es la materia prima de Podemos. Podemos es la ilusión que ha provocado una alegría plebeya, que emerge como voluntad política con pretensión de construir un punto de vista general y un horizonte de sentido para el cambio político ante la situación de crisis del régimen. Y esta ilusión ha consumado una ruptura cultural con el bipartidismo. Podemos desplaza al sistema político y muestra la vejez del sistema de partidos nacidos tras la Constitución de 1978. Aunque su proyección deberá confirmarse en las próximas citas electorales, Podemos estará de una u otra manera en el centro político durante los próximos años.

Un elemento clave, a menudo invisibilizado, para entender el desplegamiento de Podemos ha sido la experimentación tecnopolítica, la innovación política del uso de la tecnología y las herramientas para la acción colectiva. En nuestra opinión, el proyecto ha funcionado gracias a la combinación de nuevas herramientas, una masa crítica muy amplia con profundo deseo de participar en el proceso de construcción de la organización, que se articula desde una comunicación multicapa y que expresa una nueva subjetividad política que apela a la construcción del pueblo como política radical, y la centralidad de un poder constituyente.

Sea cual sea el devenir histórico del proyecto, su primer año de vida ha supuesto un objeto de estudio ineludible como fenómeno político y de comunicación. El interés académico de una fuerza que pasa de no existir a ocupar el $1^{\circ}$ puesto en gran parte de las encuestas electorales no puede pasar desapercibido. El fenómeno Podemos interesa desde una perspectiva de transformación de la esfera política española, y además suscita el interés internacional que existe por las transformaciones iniciadas por el $15 \mathrm{M}$ y su deriva constituyente-institucional.

Éstas son solo unas primeras hipótesis para un trabajo de investigación.

Barcelona, 10 de marzo 2015. 


\section{Referencias}

Rivero, J. (2014). Conversaciones con Pablo Iglesias. Madrid: Ediciones Turpial.

Toret, J., Calleja, A., Marin, O., Aragon, P., Aguilera, M., Barandarian, X., Lumbrearas, A. \& Monterde, A. (2015). Tecnopolítica y 15M: La potencia de las multitudes conectadas. Barcelona: Editorial UOC.

Aguilera M. (2015) Comunicación personal 
